\title{
Cardiac arrest after sugammadex administration in a patient with variant angina
}

\author{
Sujung Lee, Jae-Wook Jung, Eunsu Kang \\ Department of Anesthesia and Pain Medicine, Inje University Haeundae Paik Hospital, Busan, Korea
}

\section{Introduction}

Sugammadex is a modified gamma-cyclodextrin that is widely used as an antidote to rocuronium-induced neuromuscular blockade; it has been reported to be efficacious and safe. A recent study, however, reported significant complications following sugammadex administration, such as hypersensitivity and anaphylaxis. There has been only a single previous report on an occurrence of coronary spasm following sugammadex administration. Here, we present a case of unexpected severe dysrhythmia and cardiac arrest after sugammadex administration in a patient with chest pain.

\section{Case Report}

A 76-year-old male who was $169.7 \mathrm{~cm}$ tall and who weighed $65.2 \mathrm{~kg}$ was scheduled for robot-assisted radical prostatectomy under general anesthesia.

When the operation was finished, we gave him $130 \mathrm{mg}$ sugammadex (Bridion ${ }^{\circledR}$, N.V. Organon, Oss, Netherlands).

Two minutes later, sudden ventricular premature contraction (VPC) bigeminy appeared on the EKG, soon after the cardiac arrest was developed. CPR started immediately, epinephrine $1 \mathrm{mg}$ was administered. Soon after VT occured, $200 \mathrm{~J}$ of DC cardioversion was done twice. The patient was return to spontaneous circulation, but 10 minutes later VT recurred. After administration of $200 \mathrm{~J}$ of cardioversion and $2 \mathrm{~g}$ of magnesium i.v. The patient recovered directly; his EKG showed a sinus rhythm with mild tachycardia and a heart rate of $90-100$ beats $/ \mathrm{min}$.

On postoperative day 1, his CK-MB/troponin I level was increased to 15.6/8.21 ng/ml; coronary angiography was done, which showed coronary artery obstructive disease. Concentric tubular stenotic lesions in the right coronary artery and left circumflex artery, which blocked $40 \%$ and $50 \%$ of the blood flow, respectively, were observed. Also, a coronary spasm was observed on the ergonovine test, and the patient was diagnosed with variant angina [Fig. 1]. The EKG results of the VPC bigeminy obtained from the ergonovine test were similar to those obtained in the two minutes after sugammadex administration [Fig. 2]

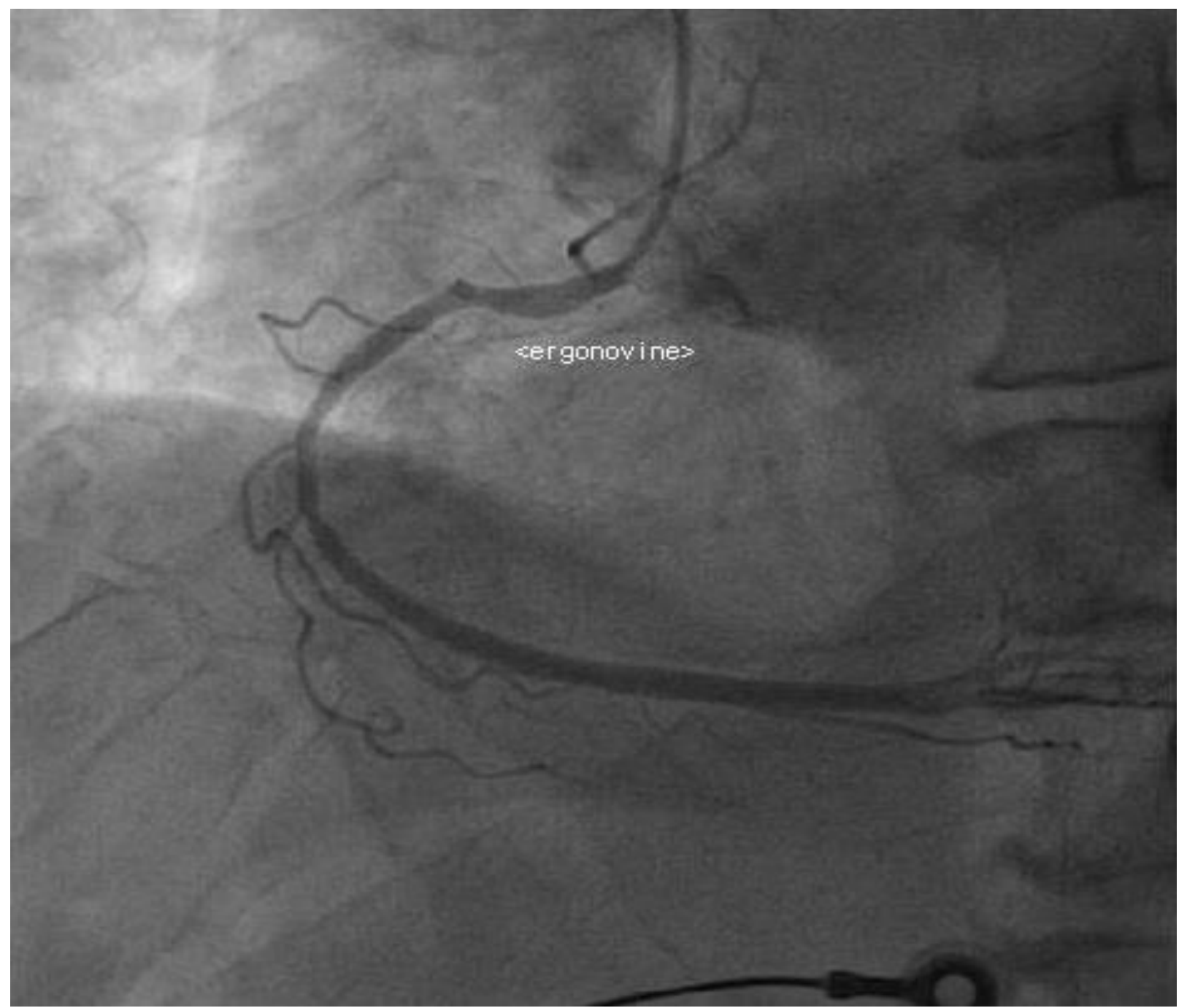

Fig. 1 Coronary spasm was observed in the ergonovine test

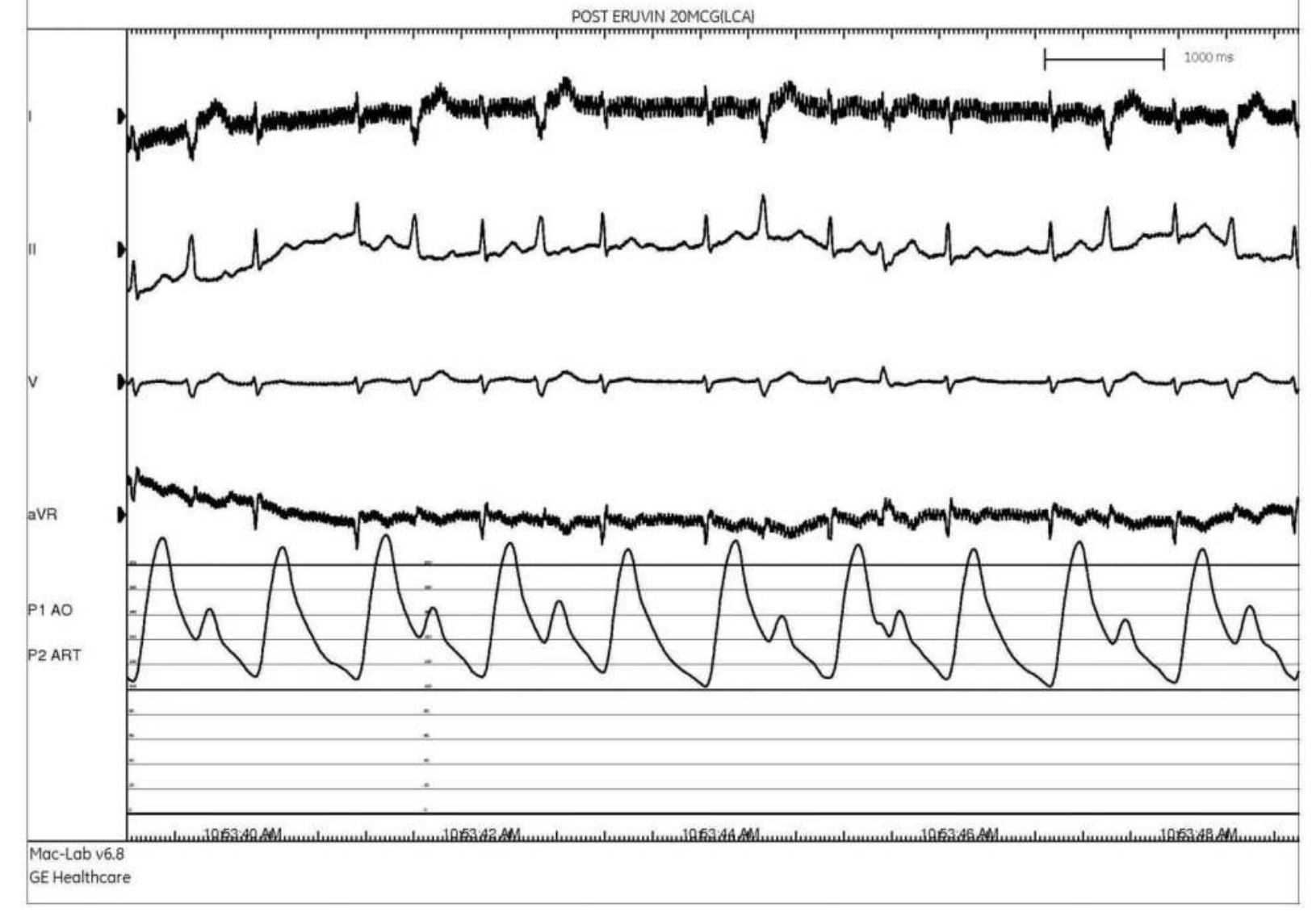

Fig. 2 VPC in the ergonovine test

On postoperative day 1, the patient's trachea was extubated; oxygen saturation was maintained well with spontaneous respiration. On postoperative day 2 , the patient's blood pressure was normal without vasopressors. The patient was transferred to the general ward on postoperative day 2. On postoperative day 8 , the patient was discharged uneventfully with normal EKG and laboratory results. After 4 weeks, the patient visited our outpatient clinic; intradermal testing was done with a 1:100 dilution of sugammadex $100 \mathrm{mg} / \mathrm{ml}$, rocuronium (1:50) and sugammadex-rocuronium complex, and the results were negative.

\section{Discussion}

In this case, the patient had three occurrences of sudden cardiac arrest after administration of sugammadex. CPR was done for about 40 minutes total; after the third CPR, the patient recovered and showed hemodynamic stability. The cause of cardiac arrest is unclear. However, coronary angiography with an ergonovine test showed variant angina postoperatively.

First, we presumed anaphylaxis to be the cause of cardiac arrest. Several cases of anaphylaxis or severe cardiac collapse after administration of sugammadex have been previously reported. However, intradermal testing with sugammadex, rocuronium, and sugammadex-rocuronium complex showed negative results. In addition, the patient had no symptoms of an allergic reaction, such as a skin rash or angioedema. Therefore, the possibility of anaphylaxis was low. Second, we considered that the cardiac arrest resulted from acute myocardial infarction accompanied by a coronary spasm.

We identified the administration of sugammadex as a more probable cause of the coronary spasm. VPC bigeminy appeared 2 minutes after sugammadex administration and severe hypotension; bradycardia occurred simultaneously. In addition, the EKG results of VPC bigeminy obtained 2 minutes after the administration of sugammadex were similar to those obtained from the ergonovine test. Therefore, we considered that the patient's severe dysrhythmia and cardiac arrest could have been triggered by sugammadex given the time course of drug administration. 